\title{
The GPS/INS Integrated Navigation Module Research and Design Based on The STM32 \\ Yang $\mathrm{Xu}^{1, \mathrm{a}}$, Peng Chen ${ }^{2, \mathrm{~b}}$ \\ ${ }^{1}$ School of Automation, Chongqing University of Posts and Telecommunications, ChongQing 400065, China; \\ ${ }^{2}$ School of Automation, Chongqing University of Posts and Telecommunications, ChongQing 400065, China; a342870@qq.com, b842620568@qq.com
}

Keywords:GPS, Integrated navigation system,Micro inertial measurement unit,STM32controller.

\begin{abstract}
This paper designs a low-cost embedded GPS/INS integrated navigation module ,using STM32 as control processing unit and the micro inertial measurement unit (MPU6050) as the sensor and GPS receiver. The GPS receiver is easily to be derived of navigation function because of the lock-loss when vehicles run in tunnels, high-rise buildings and forest environments, and this module solved this problem effectively. This paper discusses the design and implementation of hardware and software about the integrated navigation module. The experiment results show that the integrated navigation module can accurately locate the position in 20s when lost GPS signals; it has reached design requirements and has good practicality.
\end{abstract}

\section{Introduction}

Nowadays, the city traffic is becoming more and more complex. GPS can not be used continuously for city vehicle navigation due to the loss of satellite signals by obstructions from buildings, tunnels and trees etc. and it leads to low positioning accuracy[5]. In order to improve the reliability of the navigation system performance, the relevant domestic and overseas research point out that inertial navigation system (INS) and integrated navigation system (GPS/INS) navigation [1] can be used to navigate. although inertial navigation system (INS) can provide comprehensive navigation information, and fully autonomous navigation system, its error accumulate over time.Although part of the integrated navigation system (GPS / INS) meet positioning requirements, high cost makes it difficult to achieve the above in a vehicle due to the large volume. Therefore, this article designs a kind of GPS/INS integrated navigation based on STM32 module, which makes use of the stability and high precision of GPS to compensate the disadvantages of INS error increasing with time, making up for the GPS receiver with INS short-term high precision in interference or lost signal shortcomings when positioning blind area[2]. Not only positioning accurately, but also meet the requirements of car navigation by the low cost, small size,. This article introduces the hardware and software of the integrated navigation module, integrated navigation algorithm, and the experimental results.

\section{The system composition and function}

The embedded (GPS/INS) integrated navigation module includes power module, INS module, GPS module, STM32 control module, etc. Among them, the power supply module for the entire system to provide power supply; INS module and STM32 through the IIC bus communication, to provide the movement velocity and acceleration information.GPS module via a serial port and STM32 communication, to provide external auxiliary information such as position and speed of three-dimensional;STM32 control module receives the measurement information to INS and GPS, 
with the observation mode controller Kalman filtering for initial alignment and then use loose combination navigate solver, and communicate via a serial port and PC monitoring software.

The module hardware block diagram shown in Figure 1:

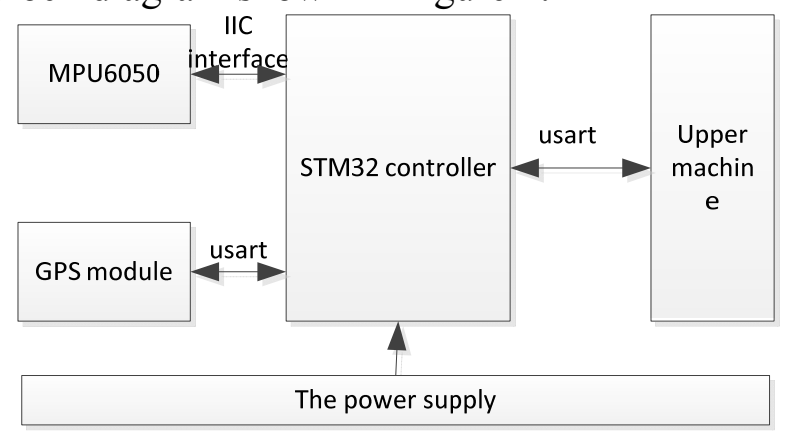

Fig. 1 integrated navigation module block diagram

\section{Software architecture and algorithms of the System}

The software design of Integrated navigation module.Integrated navigation module software includes data acquisition, system calibration and navigation solution. In the software design, the use of $\mathrm{C}$ language programming. In order to ensure the accuracy of navigation, the key issue is that the entire programming INS and GPS navigation data synchronization, you must provide the processor the same time navigation data. Since the GPS information output frequency $1 \mathrm{~Hz}$, in order to ensure the synchronization of MPU6050 data acquisition, the system uses $1 \mathrm{~Hz}$ pulse signal output from the GPS receiver as the system timing, synchronization to complete the design. System software flow chart shown in Figure 2:

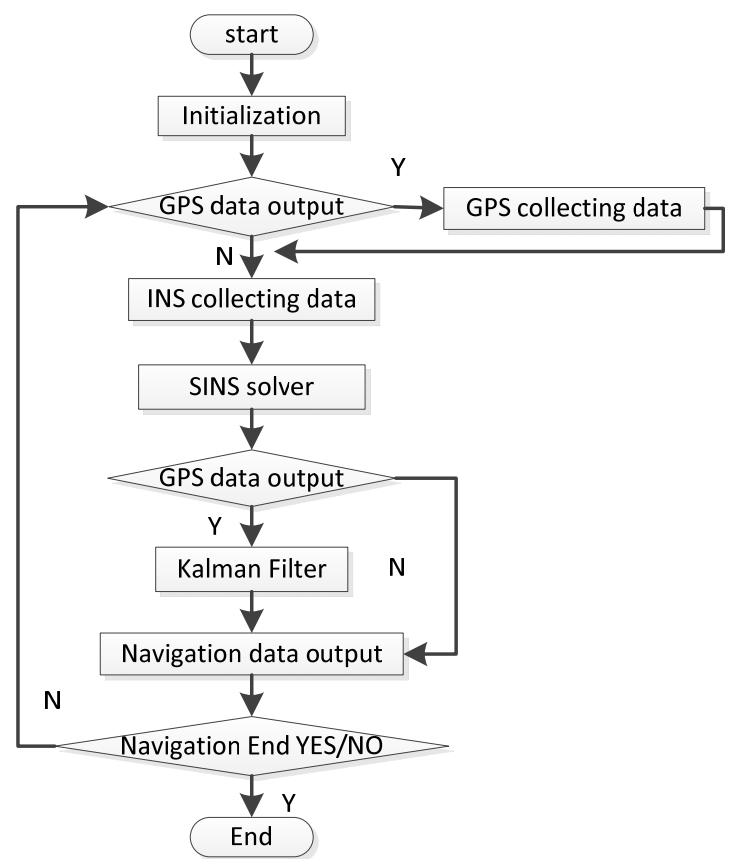

Fig. 2 System software flow chart

First, the initialization of each module, it is judged whether GPS have data output. If GPS have data output, read the GPS data and use it as the initial data of the INS.Secondly, the data acquisition module INS, and with four strap-element method solver get positioning data.Finally, once again check the GPS signal,if there is a signal, read the GPS data and used in conjunction with strapdown solver data fusion Kalman filter algorithm. if it is no signal output directly strap-down solver positioning data.

The SINS solver. SINS solver algorithm have various methods such as Euler angles, direction cosine law and quaternion. Among them, since the calculation amount and accuracy quaternion are to meet the requirements of real-time systems in general, and therefore wider application in 
practical engineering. This paper chooses four elements method.Strap-down Attitude computing consists of two parts: determined attitude matrix $\mathrm{C}_{\mathrm{n}}^{\mathrm{b}}$ and use $\mathrm{C}_{\mathrm{n}}^{\mathrm{b}}$ Solutions calculates the actual attitude angle[1], can be obtained by derivation from the carrier Differential coordinates to the four elements of the geographic coordinate system expressed as $\dot{q}(t)=\frac{1}{2} q(t) \omega_{n b}^{b}$.

$\omega_{\mathrm{nb}}^{\mathrm{b}}$ is Vector coordinates $\mathrm{b}$ relative to the geographic coordinate

$\mathrm{n}$ rotational angular velocity in $\mathrm{b}$ Projection system, and $\mathrm{q}(\mathrm{t})$ for the four elements of the vector.

Four navigation solution element method calculation shown in Figure 3:

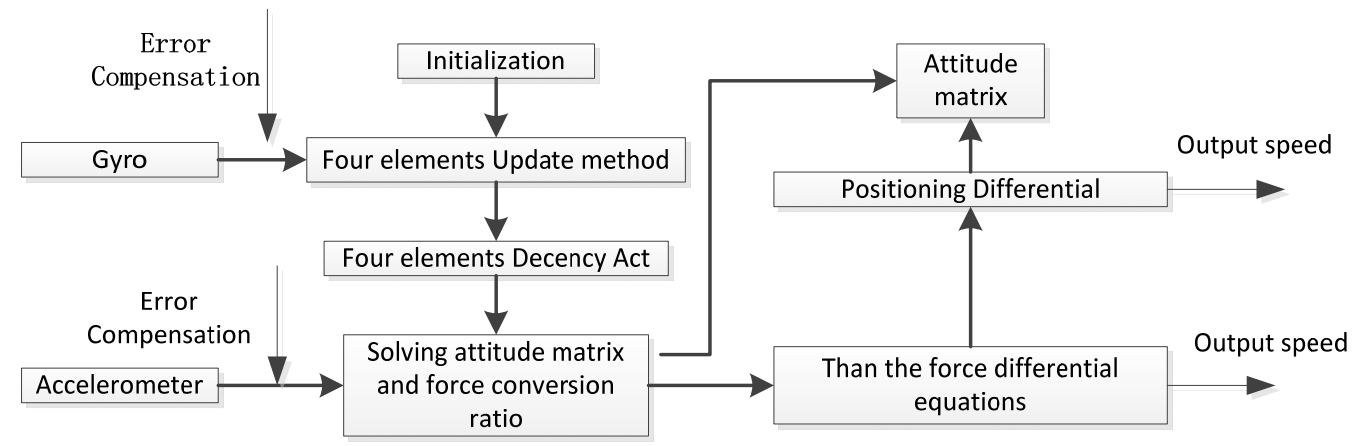

Fig. 3 SINS solver block diagram

The Navigation Algorithm. Kalman filter is a real-time status estimator in (GPS/INS) Integrated Navigation System for inertial navigation state estimation error [3], in this experiment, using indirect feedback Kalman filter structure, system state variables are:

$\mathrm{X}=[\mathrm{VE}, \mathrm{VN}, \mathrm{Vu}, \mathrm{L}, \lambda, \mathrm{h}, \Phi \mathrm{E}, \Phi \mathrm{N}, \Phi \mathrm{U}, \mathrm{dE}, \mathrm{dN}, \mathrm{dU}]$

Carrier speed is VE, VN, Vu, carrier position $\mathrm{L}, \lambda, \mathrm{h}$, platform attitude angle error is $\Phi \mathrm{E}, \Phi \mathrm{N}$, $\Phi \mathrm{U}$, gyro constant drift $\mathrm{dE}, \mathrm{dN}, \mathrm{dU}$.

System state equation is:

$$
\dot{\mathrm{X}}(\mathrm{t})=\mathrm{A}(\mathrm{t}) \mathrm{X}(\mathrm{t})+\mathrm{B}(\mathrm{t}) \mathrm{W}(\mathrm{t})
$$

$\mathrm{A}(\mathrm{t})$ is the state transition matrix, $\mathrm{W}(\mathrm{t})$ is the system noise matrix. Navigation System measurement equation is:

Observations are:

$$
\mathrm{Z}(\mathrm{t})=\mathrm{H}(\mathrm{t}) \mathrm{X}(\mathrm{t})+\mathrm{V}(\mathrm{t})
$$

$$
\mathrm{Z}(\mathrm{t})=\left[\begin{array}{c}
V i e-V g e \\
V i n-V g n \\
V i u-V g u \\
L i-L g \\
\lambda \mathrm{i}-\lambda \mathrm{g} \\
h i-h g
\end{array}\right],
$$

Vie, Vin, Viu, Li, $\lambda \mathrm{i}$, hi is the speed and position information of the inertial system solver, Vge, Vgn, Vgu, Lg, $\lambda g$, hg is the speed of the GPS location information.

Finally, usingthe optimal inertial navigation error to correct the error, including the initial alignment error, inertial measurement device error, calculation errors [4].

\section{The test of results}

In order to test the performance of embedded GPS / INS integrated navigation module, the module is installed on the smart small car, and around the school in a laboratory building outdoor experiments. First, the GPS module installed in a small car, use it to determine the initial position and the nominal trajectory. Then, the navigation module is installed for testing in a small car. The tests include acceleration, constant speed, turning, uniform four stages, a total of $120 \mathrm{~S}$. At rest, the solution vector calculated initial attitude and heading angles. The first 10 seconds is the acceleration phase, the speed of the car after accelerated $1 \mathrm{~m} / \mathrm{s}$, between $10-40$ seconds, the car speed is $1 \mathrm{~m} / \mathrm{s}$ for 
uniform operation, between 40-60 seconds the car is turn phase, between 60-100 seconds, artificially make GPS signal is lost, it can not be corrected INS system, only the INS autonomous navigation, the final 20 seconds to restore the GPS signal. In order to facilitate the observation, using Matlab graphics processing to obtain the following results (blue lines represent the measured GPS nominal trajectory, the red line represents the integrated navigation module measured track), shown in Figure 6:
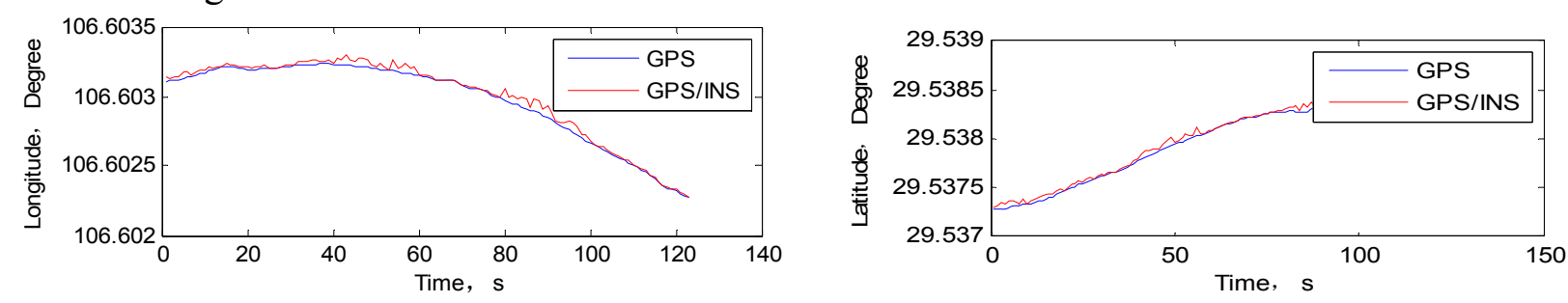

Fig. 4 positioning results

The left is a combination of GPS module and navigation module measured longitude data, the right is a combination of GPS module and navigation module measured latitude data. As can be seen from the chart, In the acceleration phase (0-10S) and turn phase (40-60S), the integrated navigation module measured latitude and longitude data compared to deviate from the nominal trajectory, which is due to lower GPS data update rate, the larger mobility when not well positioned. In uniform stage (10-40S), integrated navigation data measured latitude and longitude can track the nominal trajectory. When (60-100S) GPS signal is lost, the INS conducted autonomous navigation, it can be seen within the first integrated navigation module $20 \mathrm{~S}$ measured trajectories can be a good track nominal trajectory, but after 20 seconds was measured trajectories diverge start not a good track nominal trajectory. In the last $20 \mathrm{~s}$, because the GPS signals can be obtained, for INS correction, so that the measured trajectories navigation module, and can track the nominal trajectory.

\section{Summary}

This design of integrated navigation module uses a low-cost chip MPU6050 INS and GPS chips to STM32 processor controller module small, light weight meet the requirements of car navigation. The experiment proved that the integrated navigation system GPS signal is lost in a short time can be precise navigation.

\section{Acknowledgments}

It is a project supported by Graduate Innovative Education Key Program in Chongqing University of Posts and Telecommunication, and the project number is Y201308.

\section{References}

[1] Songlai Han, and Jinling Wang, "Quantization and Colored Noises Error Modeling for Inertial Sensors for GPS/INS Integration,” IEEE SENSORS JOURNAL, vol. 11, no. 6, June 2011.

[2] Jau-Hsiung Wang, "Intelligent MEMS INS/GPS Integration For Land Vehicle Navigation," $\mathrm{PhD}$ thesis, Calgary: Department of Geomatics Engineering, University of Calgary, 2006.

[3] Hwang D H , Oh S H , et al. Design of a low -cost atti-tude determination GPS/ INS integrated navigation sys-tem[ J] . GPS Solutions( S1080-5370), 2005, 9( 4): 294-311.

[4] Markley F Landis, Sedlak Joseph E. Kalman filter for spinning spacecraft attitude estimation[ J] . Journal ofGuidance Contorl and Dynamics (S0731 - 5090),2008, 31( 6) : 1750-1760. 
[5 ] Venckauskas A, Morkevicius N, KulikanskasK. Study of finger vein authentication algorithms for physical access control[J]. Elektronika IR Elektrotechnika, 2012 , 121 (5): $101-104$. 